\title{
Did rotating AGB stars pollute the galactic globular clusters?
}

\author{
T. Decressin ${ }^{1} \&$ C. Charbonnel ${ }^{1,2}$ \\ ${ }^{1}$ Observatoire de Genève, 51 ch des Maillettes, 1290 Sauverny, Switzerland \\ email: Thibaut.Decressin@obs.unige.ch \\ ${ }^{2}$ LA2T, OMP, CNRS UMR 5572, 14 Av. E. Belin, 31400 Toulouse, France \\ email: Corinne.Charbonnel@obs.unige.ch
}

\begin{abstract}
Stars in globular clusters exhibit abundance patterns such as the well-known O-Na and $\mathrm{Mg}-\mathrm{Al}$ anticorrelations which have no counterpart among halo stars. Whereas winds from AGB stars have been proposed as the primordial sources for these anomalies, the impact of rotation in these stars has not been studied in the GC context. To address this issue we present a model of a rotating AGB star with an initial mass of $7 \mathrm{M}_{\odot}$ and a metallicity $\mathrm{Z}=10^{-5}$. We discuss the effect of rotation on the evolutionary features, and focus on the surface abundances which can be modified by rotation, dredge-up events and hot bottom burning.
\end{abstract}

Keywords. Stars: AGB and post-AGB - globular clusters: general

\section{Stellar models}

The standard and rotating models of a $7 \mathrm{M}_{\odot}, Z=10^{-5}$ star presented here were computed with the StAREvol evolution code (Siess et al. 2000; Palacios et al. 2003).

Our treatment of rotation rests on the developments of Zahn (1992) and Maeder \& Zahn (1998). We follow simultaneously the transport of angular momentum (by meridional circulation and by vertical shear turbulence) and of the chemicals (by meridional circulation, horizontal and vertical shear) since the zero age main sequence up to the early-AGB phase. We assume an initial surface velocity of of $300 \mathrm{~km} . \mathrm{s}^{-1}$ on the ZAMS.

We use Reimers (1975) prescription to describe the mass loss rate until the end of central He burning. Then we switch to Blöcker's (1995) mass loss rate on the AGB phase. In all cases, a scaling on metallicity by a factor of $\sqrt{Z / Z_{\odot}}$ is performed.

\section{Results and comparison to globular cluster stars}

Rotation only slightly modifies the stellar structure and evolution compared to the standard case : the main sequence lifetime is almost the same, the He core burning lasts $\sim 10 \%$ longer, and the mass of the CO core at the end of central He burning is $8 \%$ higher. During the second dredge-up, primary elements are brought to the surface in the case with rotation (see below) and the metallicity thus raises up to a value of $Z=0.0017$. This results into an increase the mass loss rate which shortens the TP-AGB phase compared to the standard case.

Rotation has a large impact on the surface evolution and on the final yields of $\mathrm{O}$. During central He burning indeed primary ${ }^{16} \mathrm{O}$ diffuses out of the core, and is latter brought to the stellar surface during the second dredge-up. As can be seen in Figure 1, this results into an increase of the surface $\mathrm{O}$ abundance by about 3 orders of magnitudes. At this stage the evolution of $\mathrm{Na}$ is almost unaffected by rotation; the increase of its 
surface abundance up to the maximum value seen in globular clusters stars during the $2 \mathrm{~d}$ dredge-up reflects the conversion of the initial abundance of ${ }^{22} \mathrm{Ne}$.
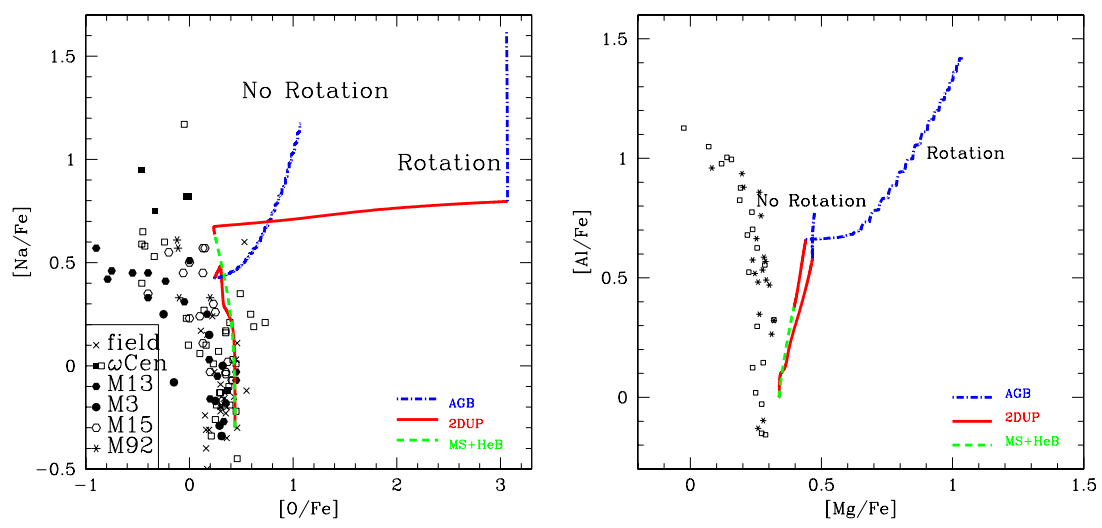

Figure 1. $\mathrm{O}-\mathrm{Na}$ and $\mathrm{Mg}-\mathrm{Al}$ anticorrelations in galactic globular clusters (data from Weiss et al. 2000 and Grundahl et al. 2002). The evolution of the surface abundances of these elements is shown for both the standard and the rotating cases for our $7 \mathrm{M}_{\odot}, Z=10^{-5}$ star. The various evolution phases are indicated.

During the AGB phase, oxygen undergoes only small destruction by hot bottom burning; this is insufficient to reverse the huge increase due to $2 \mathrm{~d}$ dredge-up. Meanwhile sodium is enhanced thanks to hot bottom burning and 3d dredge-up; its surface abundance reaches values larger than the ones observed in globular clusters stars.

The abundances of heavier elements like magnesium or aluminum are less affected by rotation. In both the standard and the rotating cases a production of both elements is found. Moreover, the relative abundances of magnesium isotopes found by Yong et al. (2003) are not reproduced by neither our standard nor our rotating models.

\section{Conclusions}

Due to the effect of rotation, massive metal-poor AGB stars build up an O-Na correlation, with an extreme enrichment in $\mathrm{O}$, as well as a $\mathrm{Mg}$-Al correlation and an important increase in $\mathrm{C}+\mathrm{N}+\mathrm{O}$ (not shown here). All these predictions disagree with the observations (see Sneden and Charbonnel, these proceedings). It seems thus that these objects are not responsible for the abundances anomalies observed in globular cluster stars.

\section{References}

Blöcker T., 1995, A\&A, 297, 727 Tables, 40, 283

Herwig F., 2000, $A \& A, 360,952$

Grundahl F., Briley M., Nissen P.E., \& Feltzing S., 2002, A\&A, 385, L14

Maeder A. \& Zahn J.-P., 1998, A\&A, 334, 1000

Meynet G. \& Maeder A., 2002, AESA, 381, L25

Palacios A., Talon S., Charbonnel C., \& Forestini M., 2003, A\&A A, 399, 603

Reimers D., 1975, Mem. Soc. Roy. Sci. Liège, 6th Ser., 8, 369

Siess, L., Dufour, E., \& Forestini, M., 2000, A\& $A$, 358, 593

Weiss A., Denissenkov P.A., \& Charbonnel, C., 2000, A\&A, 356, 181

Yong D., Lambert D.L., \& Ivans I.I., 2003, ApJ, 599, 1357

Zahn J.-P., 1992, A\&A, 265, 115 